> La biologie intégrative connaît actuellement un profond renouveau avec la montée en puissance de la biologie systémique qui explore la logique du vivant et de la biologie synthétique qui l'exploite. <

\section{Biologie \\ synthétique \\ et intégrative}

François Képès

\section{Le renouveau de la biologie}

La biologie entre dans une ère où les percées fondamentales viendront des couplages entre théorie et expérimentation, et entre efforts analytiques et synthétiques. La biologie moléculaire est née avec l'apport décisif des physiciens, puis s'est institutionnalisée dans les années 1970. II en va de même du renouveau de la biologie intégrative dans les années 2000 . L'actuelle révolution s'annonce de même ampleur que la précédente. Elle bouleverse le processus de la découverte en biologie et l'ingénierie de la biologie. Selon la terminologie internationale, ces deux aspects relèvent respectivement de la biologie systémique et de la biologie synthétique.

Peu d'éléments de notre vie échapperont aux conséquences de cette révolution, de la médecine jusqu'à la production d'énergie en passant par les biomatériaux. L'ampleur de ces bouleversements s'apparentera à celle des sciences de l'information depuis 30 ans ou de la chimie de synthèse un siècle plus tôt.

La biologie systémique tente de comprendre les comportements de réseaux biologiques d'interaction, en particulier leurs aspects dynamiques et leurs développements spatiaux. Elle recourt typiquement à l'import transdisciplinaire des concepts et au couplage entre théorie et expérimentation. La biologie systémique offre accès à la logique du vivant (life's design principles). II est naturel d'ensuite appliquer ces principes pour synthétiser des systèmes biologiques. C'est l'objectif de la biologie de synthèse, dite "synthétique », l'ingénierie de la biologie : la conception rationnelle et la construction de systèmes complexes basés sur ou inspirés par le vivant mais dotés de fonctions absentes dans la nature. Cette perspective d'ingénierie peut s'appliquer à toutes les échelles des structures biologiques - des molécules individuelles aux cellules, tissus et organismes. Ici, la nouveauté réside dans l'attitude scientifique, plus

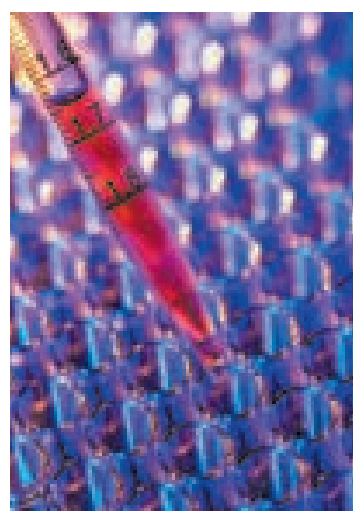

Programme d'épigénomique, Genopole ${ }^{\circledR}$, CNRS, Université d'Évry, 523, Terrasses de l'Agora, 91034 Évry, France francois.kepes@ epigenomique.genopole.fr

synthétique qu'analytique, et donc dans le typage des questions et réponses scientifiques.

L'exemple du rythme circadien permettra d'illustrer la complémentarité entre biologies systémique et synthétique. Le rythme circadien est celui qui règle nos jours et nuits selon un cycle proche de 24 heures. Les généticiens ont découvert, il y a longtemps, les gènes et protéines impliqués dans ce rythme biologique. Cependant, l'explication scientifique du phénomène cyclique n'est pas venue des généticiens mais des modélisateurs comme Albert Goldbeter à l'Université libre de Bruxelles (Belgique) qui ont montré comment les produits de ces gènes contribuent collectivement à un oscillateur qui règle le rythme circadien [1]. C'est un joli cas de succès scientifique de l'approche par la biologie systémique. Imaginons maintenant qu'une entreprise confie à son ingénieur biologiste le soin de réaliser une horloge biocompatible rythmant une pompe à insuline, à implanter dans le corps d'un diabétique. Cet ingénieur, ayant compris les principes de l'oscillateur circadien, cherchera à les réutiliser dans un contexte différent et avec des paramètres altérés (par exemple, son cahier des charges spécifie un cycle d'une heure plutôt que 24 heures). II va mettre sur pied un modèle mathématique inspiré de celui du rythme circadien (modélisation), le «faire tourner» avec l'ordinateur (simulation), modifier des paramètres (investigation) jusqu'à respecter le cahier des charges. II va ensuite réaliser les constructions nécessaires par génie génétique, puis mesurer les propriétés du système ainsi réalisé. Supposons qu'il mesure, entre autres propriétés, un cycle de deux heures. Ce résultat expérimental va lui permettre 
de re-calibrer son modèle mathématique plus près du résultat souhaité d'une heure. L'investigation par simulation va lui suggérer de rendre plus instable l'une des protéines de l'oscillateur. II va retourner à la paillasse, modifier le code de cette protéine dans cette intention, puis mesurer à nouveau les propriétés du système, maintenant plus proches du but. On le voit, il s'agit d'instituer une spirale d'amélioration itérative allant du modèle et sa simulation, via la construction des objets biologiques, à la mesure de leurs propriétés. Cela est une des caractéristiques fortes de cette approche synthétique.

\section{Enjeux et perspectives}

Deux analogies permettent d'appréhender l'immense potentiel de la biologie synthétique.

1. La chimie organique a débuté comme une science analytique, visant à purifier et caractériser des molécules naturelles. Grâce à l'accroissement des connaissances sur la structure et réactivité des molécules naturelles, la chimie synthétique s'est ensuite développée comme un complément de la chimie analytique. Initialement, la chimie de synthèse permettait de confirmer les résultats de la chimie analytique ou de produire plus de matériel à fins d'analyse. Cependant, les capacités nécessaires pour la synthèse d'une molécule, naturelle ou non, sont les mêmes. Cela a donc fini par mener vers la synthèse de structures absentes dans la Nature, dont certaines imitent ou améliorent des propriétés de molécules naturelles.

Or, un siècle plus tard, la biologie a atteint le stade des méthodes en «-omiques » qui couronnent ses capacités analytiques. Aussi, les connaissances accumulées commencentelles à être suffisantes pour entrer dans l'époque synthétique. Par analogie à la chimie de synthèse, la biologie synthétique ne se contente pas d'expliquer ou reproduire le comportement de systèmes naturels. Son objectif est plutôt de construire de nouveaux systèmes biologiques fondés sur les principes de design observés dans la Nature, mais avec des propriétés augmentées, étendues et contrôlables. La complexité de cet objectif de design rend impérative une approche d'ingénierie ancrée dans la modélisation. Faire «tourner le modèle » dans la tête du biologiste est généralement suffisant quand il s'agit d'exprimer fortement un gène dans un hôte cellulaire par exemple, comme il est usuel en génie génétique. En revanche, étant donnée la non-linéarité des réponses biochimiques, la modélisation mathématique devient nécessaire pour concevoir un dispositif régulatoire ou métabolique impliquant plusieurs composants. Après la conception du dispositif, l'étape de sa construction fait appel aux outils classiques du génie génétique, si ce n'est que l'accent méthodologique est placé sur la systématisation et la standardisation des procédés et des composants de base.

2. Cependant, les processus de découverte et d'industrialisation se sont accélérés depuis un siècle, et il est probable que le développement de la biologie synthétique ressemble plutôt par sa dynamique à celui de l'industrie informatique. Son stade actuel s'apparente à celui des tous premiers jours de l'industrie informatique. En effet, la biologie synthétique peut changer fondamentalement notre approche de certaines technologiesclés, en particulier en médecine et en bioproduction. Pourtant, il est trop tôt pour prédire où se situeront les applications les plus importantes, même si la biologie synthétique a déjà concrétisé des succès; c'est ainsi par exemple que 400000 patients atteints de Sida ou d'hépatite sont suivis chaque année grâce à la technologie VERSANTTM [2, 3] de Bayer Diagnostics.

$\varepsilon$ n tout cas, la biologie synthétique créera les technologies génériques servant à exploiter les outils et processus bio-inspirés à des fins industrielles et commerciales. Il est probable que la connaissance provenant du domaine public sera très rapidement incorporée dans un vaste ensemble de «solutions » technologiques, créant ainsi une arène dans laquelle se jouera le contrôle de la propriété intellectuelle.

La biologie synthétique est un domaine naissant et il n'existe pas aujourd'hui d'effort global et systématique pour en coordonner les développements. La majeure partie de cette recherche a jusqu'à présent été menée par quelques groupes pionniers aux États-Unis. Cependant, l'effet de concentration, annonciateur d'une accélération de l'industrialisation, est symbolisé par le fait que le plus gros financement d'un projet en biologie vient de s'opérer justement en biologie synthétique : il s'agit d'un projet dirigé par Jay Keasling à Caltech, qui a rassemblé environ un milliard de dollars, dont la moitié fournie par British Petroleum.

\section{Émergence de la biologie synthétique}

Les raisons de l'actuelle émergence sont d'ordres technologique et fondamental.

Sur le plan fondamental, il est devenu clair qu'une compréhension du fonctionnement de la cellule ou de l'organisme requiert plus qu'une simple liste des composants telle que le livrent certaines «-omiques» (génomique, transcriptomique, protéomique, métabolomique, etc.). II s'agit de comprendre comment les gènes, les protéines et autres biomolécules interagissent pour former des modules et circuits fonctionnels, et d'en avoir une vision causale et dynamique. Cette compréhension, qui progresse par les approches dites de biologie systémique, fournit les outils conceptuels requis ensuite pour le design et la construction rationnelle de circuits biologiques, dans le cadre de la biologie synthétique.

Sur le plan technologique, les méthodes standard de la biochimie, de la biophysique et de la biologie moléculaire, qui permettent de modifier les protéines et de réarranger l'information génétique, ont progressé au point d'être utilisables pour le design des cartes d'interaction dans les cellules vivantes. Point essentiel pour le futur, la longueur des brins 
d'ADN que l'on sait synthétiser d'un seul tenant progresse exponentiellement d'année en année. II est déjà possible de produire des séquences spécifiées d'une longueur comparable à celle d'un génome viral. Aussi sera-t-il bientôt possible de synthétiser entièrement des génomes bactériens, point de départ pour la création de certaines cellules artificielles.

\section{Potentiel et applications de la biologie synthétique}

Les applications de la biologie synthétique traversent de nombreux champs disciplinaires, depuis la médecine jusqu'à la production d'énergie. Ses domaines potentiels incluent bioproduction, biosenseurs, biomatériaux, nanothérapeutique, biosécurité.

Par exemple, des micro-organismes pourraient être conçus pour produire des composés pharmaceutiques qui représentent un défi irréaliste pour les méthodes existantes de synthèse chimique ou microbiologique. S'il est vrai que plusieurs composés pharmaceutiques sont déjà produits en biotechnologie par des organismes génétiquement modifiés, il est non moins clair que la capacité de réaliser des voies régulées et complexes de synthèse augmenterait considérablement le répertoire de composés concevables. Un exemple actuel en est la synthèse par voie de biologie synthétique, à coût très réduit, de l'artémisine, médicament anti-malaria, dans le laboratoire de Jay Keasling déjà mentionné $[4,5]$.

Des micro-dispositifs biologiques fondés sur l'assemblage modulaire d'ADN, de lipides et de protéines pourraient agir dans le corps humain pour détecter et corriger des pathologies à leur stade précoce, pour réparer ou régénérer des tissus. Ainsi, la biologie synthétique offrirait les outils pour une intervention médicale au niveau moléculaire, prévenant donc le recours aux pratiques plus grossières de la chirurgie et de la pharmacopée. Si l'aspect thérapeutique reste aujourd'hui à l'état de promesse, l'aspect diagnostique offre des avancées concrètes, la technologie VERSANTTM en étant un exemple déjà mentionné [2, 3].

De la même manière que ces organismes modifiés pourraient fournir des nouveaux médicaments, ils pourraient être conçus pour produire des matériaux utiles - par exemple des matières plastiques biodégradables - à partir d'éléments bruts, renouvelables et de faible coût, ou pour convertir ces sources en carburants comme l'hydrogène ou le méthanol. Ceci pourrait rendre l'industrie chimique plus «verte ». Par exemple l'hydrogène pourrait être produit à partir d'énergie solaire par des bactéries se divisant bien plus vite que les algues qui réalisent naturellement cette photo-production. La capacité des systèmes biologiques à contrôler la structure des matériaux au niveau moléculaire pourrait ouvrir la voie à des matériaux dotés de propriétés nouvelles et améliorées, ou à des machines ou circuits électroniques nano-structurés.

Enfin, des systèmes biologiques synthétiques pourraient être conçus comme détecteurs autonomes et sensibles de polluants, explosifs, matériel de guerre biologique. Un projet présenté à la compétition internationale iGEM en 2006 en est un exemple: il introduisait dans des bactéries la capacité à changer macroscopiquement la couleur de leurs colonies en présence de doses dangereuses d'arsenic [6] ; I'application immédiate en était la détection à bas coût d'arsenic dans les puits d'eau contaminés des villages au Bangladesh. Cette capacité de détection pourrait ultérieurement être couplée à celle d'inactivation des substances dangereuses ainsi repérées.

\section{Contenus scientifiques de la biologie synthétique}

Il existe déjà plusieurs biologies synthétiques, quoique le terme ne se soit répandu que récemment. On peut notamment distinguer quatre démarches.

1. La reproduction du comportement émergent de la biologie naturelle par assemblage de molécules non naturelles (par exemple [7-9]). Cette première approche, très intéressante, nécessite plus d'investissement initial que les autres, ainsi que la présence d'autres disciplines, au plus haut niveau (chimie synthétique, microfluidique, etc.).

2. L'ingénierie efficace de circuits génétiques artificiels au comportement spécifié, par assemblage de «briques » réutilisables d'origine naturelle, à l'instar des composants d'un circuit électronique (par exemple $[10,11]$ ). Cette seconde approche présente un intérêt direct au plan du génie biotechnologique, mais seulement indirect au plan fondamental.

3. La cellule minimale (par exemple $[12,13]$ ). II s'agit de réduire les génomes bactériens à une taille minimale compatible avec la croissance. Le travail est considérable, car les gènes sont inactivés à l'unité ou par petits groupes. L'intérêt global est faible au plan fondamental par défaut d'hypothèse sous-jacente. II est limité au plan appliqué à la création d'une plate-forme, un chassis, plus simple que la version naturelle, pouvant servir à construire des applications biotechnologiques.

4. La reconstruction du vivant, avec éventuellement l'appoint de l'évolution accélérée (par exemple [14, 15]). Au plan fondamental, reconstruire autrement un processus biologique commun à toute vie sur terre (voie métabolique, code génétique, architecture chromosomique) permet d'apprendre si le processus naturel résulte de contraintes d'ordre physicochimique, ou d'un accident gelé de l'évolution. Au plan appliqué, cette approche permet d'améliorer la bioproduction.

\section{Éducation-formation-enseignement}

La nature profondément multidisciplinaire de la biologie synthétique, parce que les savoirs n'y sont pas juxtaposés mais synergiques, impose des initiatives éducatives à tous les niveaux, de l'étudiant de Mastère au chercheur 
chevronné. II s'agit de créer les compétences et la culture commune pour l'épanouissement de ce domaine. À long terme, il serait bon de créer un nouveau type de scientifiques qui soient familiarisés à la fois avec la biologie et les méthodes de l'ingénieur, tout en disposant de compétences complémentaires en chimie, ou nanotechnologie, ou dans les sciences de l'information et du contrôle. Cela impliquera d'intégrer les concepts de la biologie synthétique au cœur de certaines filières standard de l'enseignement supérieur. Le nouveau concours annuel international des machines créées génétiquement, «iGEM», est un autre élément dans l'univers éducatif de la biologie synthétique qui a la vertu d'attirer de jeunes étudiants talentueux vers la biologie $[16,17]$.

\section{Sécurité et éthique}

La biologie synthétique, dans son volet vivant, n'offre pas plus de dangers que la technique qu'elle met en œuvre pour manipuler le vivant, donc le génie génétique, qui fit l'objet d'un moratoire levé depuis plus de 30 ans. Comme précédemment, il reste possible de détourner le génie génétique vers des fins agressives. Cependant, cette possibilité n'est ni nouvelle, ni spécifique à la biologie synthétique. En outre, certains mécanismes existent pour déceler et refuser les commandes des apprentis sorciers auprès des compagnies offrant un service de synthèse d'ADN à façon. Par ailleurs, la chimie synthétique a une tradition plus ancienne que la biologie synthétique et a montré son intérêt et sa générale innocuité. Enfin, ce type de projet est généralement reconnu comme ne soulevant pas de problème éthique, mais nécessitant une éducation du public. Il est effectivement important d'éduquer le public d'emblée sur les questions d'éthique et de sécurité liées à la biologie synthétique, afin que les développements futurs s'opèrent dans des conditions de confiance citoyenne. $\diamond$

\section{SUMMARY}

Synthetic and integrative biology

Integrative biology currently undergoes a deep renewal as we witness the increasing influence of systems biology, which explores life's logic, and of synthetic biology, which exploits it. $\diamond$

\section{RÉFÉRENCES}

1. Leloup JC, Goldbeter A. Modeling the circadian clock: from molecular mechanism to physiological disorders. Bioessays 2008; 30:590-600.

2. Elbeik T, Surtihadi J, Destree M, et al. Multicenter evaluation of the performance characteristics of the bayer VERSANT HCV RNA 3.0 assay (bDNA). J Clin Microbiol $2004 ; 42: 563-9$.

3. Elbeik T, Markowitz N, Nassos P, et al. Simultaneous runs of the Bayer VERSANT HIV- 1 version 3.0 and HCV bDNA version 3.0 quantitative assays on the system 340 platform provide reliable quantitation and improved work flow. J Clin Microbiol $2004 ; 42: 3120-7$.

4. Khosla C, Keasling JD. Metabolic engineering for drug discovery and development. Nat Rev Drug Discov $2003 ; 2$ : 1019-25.

5. Keasling JD. Synthetic biology for synthetic chemistry. ACS Chem Biol 2008 ; 3: 64-76.

6. http://parts2.mit.edu/wiki/index.php/Arsenic_Biosensor

7. Luisi PL, Ferri F, Stano P. Approaches to semi-synthetic minimal cells: a review. Naturwissenschaften 2006 ; $93: 1-13$.

8. Kaneko K. Question 8: from a set of chemical reactions to reproducing cells. Orig Life Evol Biosph 2007 ; 37 : 449-53.

9. http://www.istpace.org/index.html

10. Yokobayashi Y, Weiss R, Arnold FH. Directed evolution of a genetic circuit. Proc Natl Acad Sci USA 2002 ; 99 : 16587-91.

11. Elowitz $M B$, Leibler $S$. A synthetic oscillatory network of transcriptional regulators. Nature $2000 ; 403: 335-8$.

12. Hutchison CA, Peterson SN, Gill SR, et al. Global transposon mutagenesis and a minimal Mycoplasma genome. Science $1999 ; 286: 2165-9$.

13. Pósfai $G$, Plunkett $G 3^{\text {rd }}$, Fehér $T$, et al. Emergent properties of reduced-genome Escherichia coli. Science $2006 ; 312$ : 1044-6.

14. Pezo V, Metzgar D, Hendrickson TL, et al. Artificially ambiguous genetic code confers growth yield advantage. Proc Natl Acad Sci USA 2004 ; 101 : 8593-7.

15. Képès F. Le modèle solénoïde des chromosomes. Biofutur 2007 ; 282 : 48-51.

16. Peccoud J, Coulombel L. Une compétition de biologie synthétique ou comment créer l'eau de $\varepsilon$. coli et les nano-barbies. Med Sci (Paris) $2007 ; 23: 551-2$.

17. Bikard $D$, Képès $F$. Succès de la première équipe française lors de la compétition iGEM de biologie synthétique. Med Sci (Paris) 2008 ; 24 : 541-4.

\section{SITES INTERNET}

http://www.epigenomique.genopole.fr/index.php?n=Workgroups. NewSynBio

Site français de vulgarisation sur la biologie synthétique: http://www.biologiesynthetique.fr

Site de la communauté américaine de biologie synthétique : http://www.syntheticbiology.com

Site officiel de la compétition iGEM:

http://www.igem.org

http://www.europe.igem.org
TIRÉS À PART

F. Képès 